\title{
Benefits of ESA Gravity-Related Hands-on Programmes for University Students' Careers
}

\author{
Natacha Callens ${ }^{1} \cdot$ Lily Ha $^{2} \cdot$ Piero Galeone ${ }^{2}$
}

Received: 29 January 2016 / Accepted: 5 May 2016 / Published online: 21 May 2016

(C) The Author(s) 2016. This article is published with open access at Springerlink.com

\begin{abstract}
The Education Office of the European Space Agency (ESA) offers university students, from ESA Member and Cooperating States, the opportunity to perform investigations in physical sciences, life sciences, and technology, under different gravity conditions through three educational programmes. The "Fly Your Thesis!" (FYT) programme makes use of parabolic flights and the "Drop Your Thesis!" (DYT) programme utilizes a drop tower as microgravity carriers, while the "Spin Your Thesis!" (SYT) programme uses a large centrifuge to create hypergravity. To date, more than hundred university students had the chance to participate in the design, development, and performance of one or more experiments during dedicated campaigns. In the following paper, we examine demographics of past participants of the ESA Education Office gravity-related opportunities over the past seven years and evaluate the benefits of these educational programmes for the participants' studies and careers. Student teams that participated in one of the programmes between 2009 and 2013 were contacted to fill in a questionnaire. The feedback from the students
\end{abstract}

This article belongs to the Topical Collection: Advances in Gravityrelated Phenomena in Biological, Chemical and Physical Systems Guest Editors: Valentina Shevtsova, Ruth Hemmersbach

Natacha Callens

natacha.callens@esa.int

1 European Space Agency (ESA), Education and Knowledge Management Office, REDU, 1 Place de l'ESA, 6890

Redu, Belgium

2 European Space Agency (ESA), Education and Knowledge Management Office, ESTEC, Keplerlaan 1, 2200 AG Noordwijk, The Netherlands demonstrate significant benefits extending far beyond the primary educational objectives of these programmes.

Keywords Microgravity $\cdot$ Hypergravity $\cdot$ Parabolic flights $\cdot$ Drop tower $\cdot$ Centrifuge $\cdot$ Education

\section{Introduction}

The ESA ${ }^{1}$ Education Office was created in 1998 with the purpose of motivating young people from ESA Member and Cooperating States to study science, engineering, mathematics, and technology subjects, and to ensure a better qualified workforce for ESA and the European space sector in the future. To achieve this, the ESA Education Office supports several activities that provide university students with opportunities for practical experience and transfer of knowledge through direct interaction with space professionals. Through the ESA Education Office hands-on programme opportunities, university students can develop instruments and platforms for microsatellites (Bruzzi et al. 2014), participate in the full cycle of the development of a pico-satellite (Galeone et al. 2014) and in its operations, or develop and operate sub-orbital and stratospheric flight experiments on sounding rockets and high altitude balloons (Callens et al. 2013). Additionally, students can conduct research experiments using microgravity and hypergravity carriers. The Fly Your Thesis! (FYT) programme features parabolic flights aboard the A310 ZeroG aircraft, operated by Novespace ${ }^{2}$ (Pletser and Harrod 2014), from Bordeaux-Mérignac in France, the Drop Your Thesis! (DYT) programme makes use of the Bremen Drop Tower, operated by ZARM FAB ${ }^{3}$, in

\footnotetext{
${ }^{1}$ www.esa.int

${ }^{2}$ www.novespace.fr

${ }^{3}$ www.zarm.uni-bremen.de
} 
Fig. 1 Distribution of university students per experiment campaign and per year. Each number of team members block represents a team with the

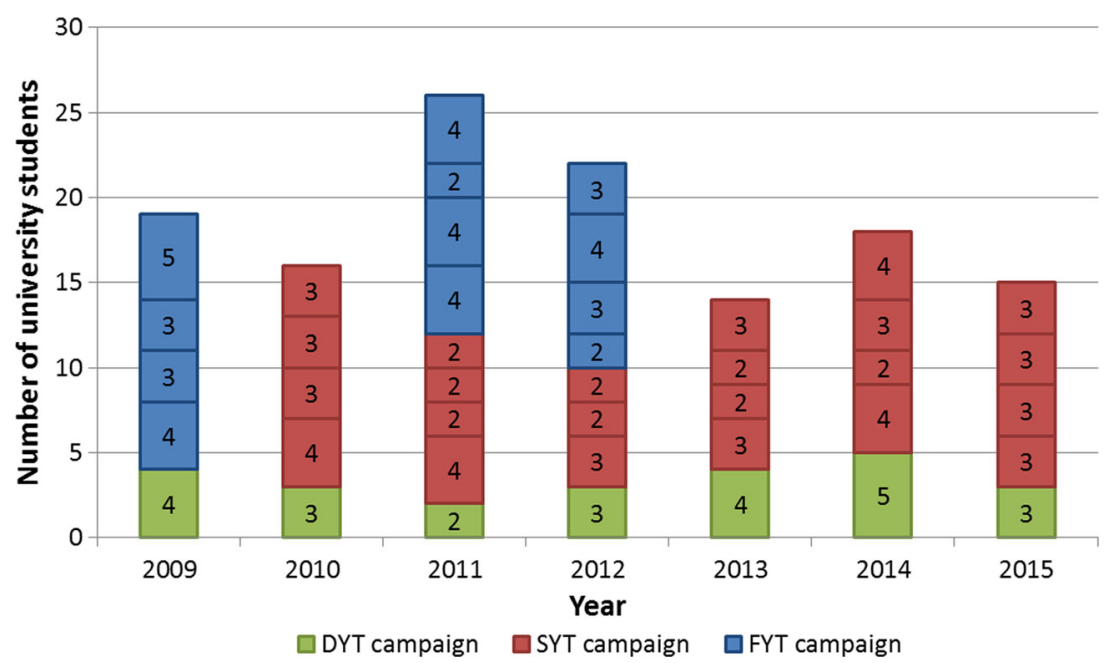

Student Participant Demographics

Bremen, Germany (von Kampen et al. 2006), and the Spin Your Thesis! (SYT) programme utilizes ESA's Large Diameter Centrifuge (LDC) of the Life, Physical Sciences and Life Support Laboratory ${ }^{4}$ at ESTEC (van Loon et al. 2008) in The Netherlands (Emma et al. 2008; Callens and Galeone 2014).

The general application process includes: formation of a student team, generally composed of two to four students; identification of an endorsing professor from their university and submission of an experiment proposal. Since 2009, the ESA Education Office has organised 16 microgravity and hypergravity experiment campaigns, 42 teams have been selected, 7 for DYT, 23 for SYT and 12 for FYT including 130 university students (Fig. 1) from 39 different universities from Europe and Canada. Students were offered the opportunity to design, build, and perform their experiment during a dedicated campaign. In the programmes' history, one team only was not able to complete the preparation of their experiment in time for a campaign. During the development of their experiments, all the student teams were provided with technical support by experts from the microgravity or hypergravity carrier, and financial support by the ESA Education Office. Furthermore, 27 teams took advantage of the scientific support offered by volunteer mentors from the European Low Gravity Research Association (ELGRA). ${ }^{5}$

One cycle, from the selection announcement to the submission of the final reports, takes approximately one year for the SYT and DYT programmes, and about one and a half years for the FYT programme. After providing their final report, students are encouraged to present their project at international conferences and to publish their results in renowned journals.

\footnotetext{
$\overline{{ }^{4} \text { www.esa.int/OOr_Activities/Space_Engineering_Technology/Life }}$ Physical_Sciences_and_Life_Support_Laboratory

${ }^{5}$ www.elgra.org
}

The participating students were $66 \%$ male and $34 \%$ female. Amongst the group, 18 different nationalities were represented: 16 ESA Member States, 1 European Cooperating State (SK) and 1 Associate Member State (CA) (Fig. 2).

The level of study of the students at time of their participation was $48 \%$ Doctorate, $32 \%$ Master and $20 \%$ Bachelor. At time of application, ages ranged from 19 to 31 years old, with an average of 24 years and a standard deviation of 2.6 years.

Approximately $80 \%$ of the participating students had a scientific background (around $60 \%$ in physical sciences and $40 \%$ in life sciences) and the other $20 \%$ an engineering background (Fig. 3).

\section{Experiments}

A variety of fields were covered by the student experiments (Fig. 4) ranging from fluid dynamics and astrophysics, to biology and human physiology. Around $56 \%$ of the experiments were in the field of physical sciences, $39 \%$ in life sciences and $5 \%$ in technology. Experiments were performed using laboratory-type equipment, as well as specific hardware developed by the students at their universities. The majority of the teams performed quantitative experiments to measure phenomena in microgravity or hypergravity and compare, when possible, with control results obtained at $1 \mathrm{~g}$. Some examples of student experiments are presented in the following sections to show the diversity of the projects and quality of their results. All final reports can be found on the ESA Education Office website. ${ }^{6}$ Eight students participated in more than one experiment campaign using the same or a different microgravity/hypergravity carrier. These students

\footnotetext{
$6_{\text {www.esa.int/Education }}$
} 
Fig. 2 Distribution of nationalities (in ISO codes) of participating students

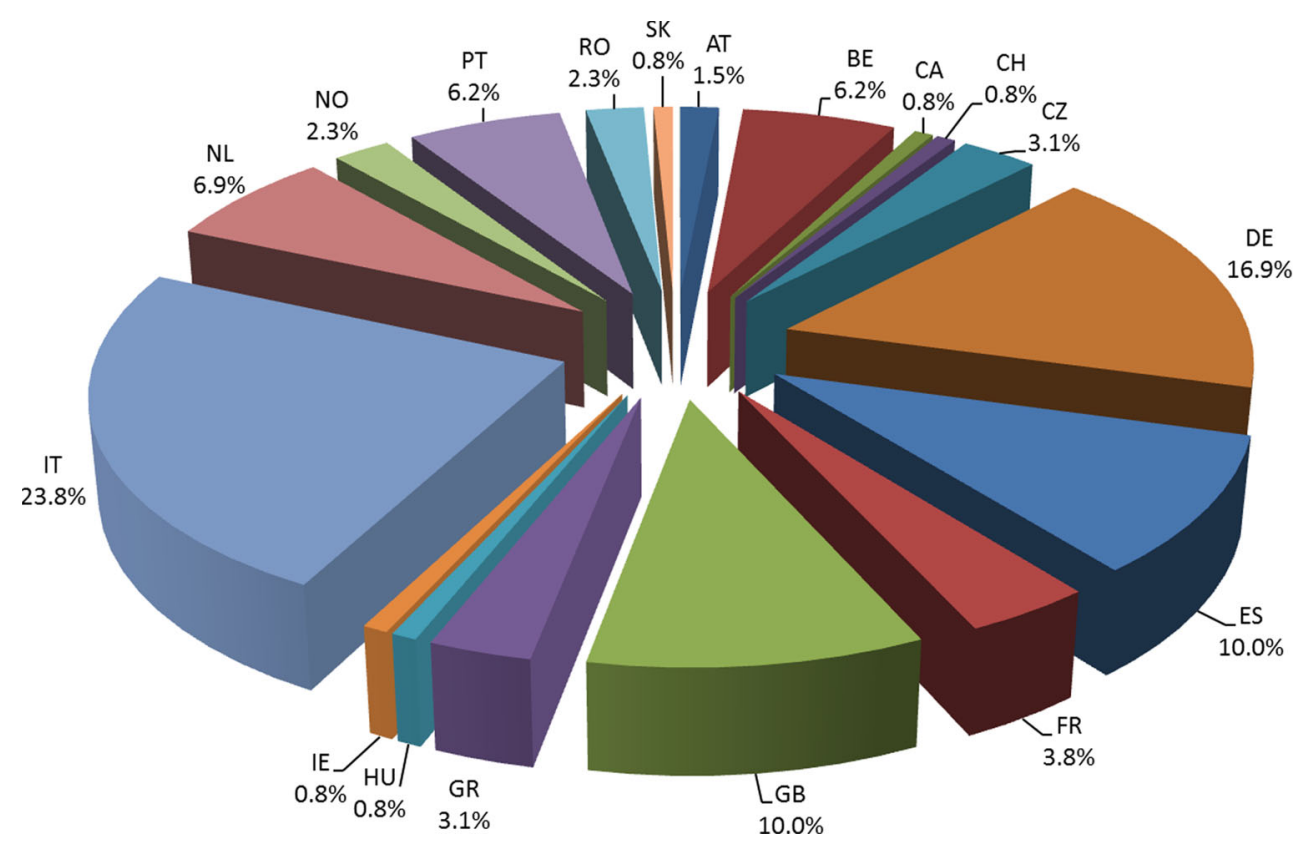

gathered additional results for the same project or performed a new experiment for a different project.

\section{“Fly Your Thesis!” Programme}

The "Fly Your Thesis!" programme offers Master and PhD students the opportunity to participate in ESA's Parabolic Flight Campaigns. The campaign consists of three flights with 30 parabolas each, providing about 20 s of weightlessness conditions per parabola at levels of reduced gravity on the order of $10^{-2} \mathrm{~g}$ (Callens et al. 2009). During the campaigns, students work in close contact with well experienced European scientists and can carry out their research. After a three year hiatus from 2013 to 2015, a new cycle of the FYT programme has been launched and a parabolic flight campaign is planned for Autumn 2016 onboard the "new" A310 ZERO-G aircraft (Pletser et al. 2015). This microgravity carrier allows university students to experience weightlessness, while having direct access to their experiment for operations during and between parabolas.
Fig. 3 Distribution of the background of the participating students

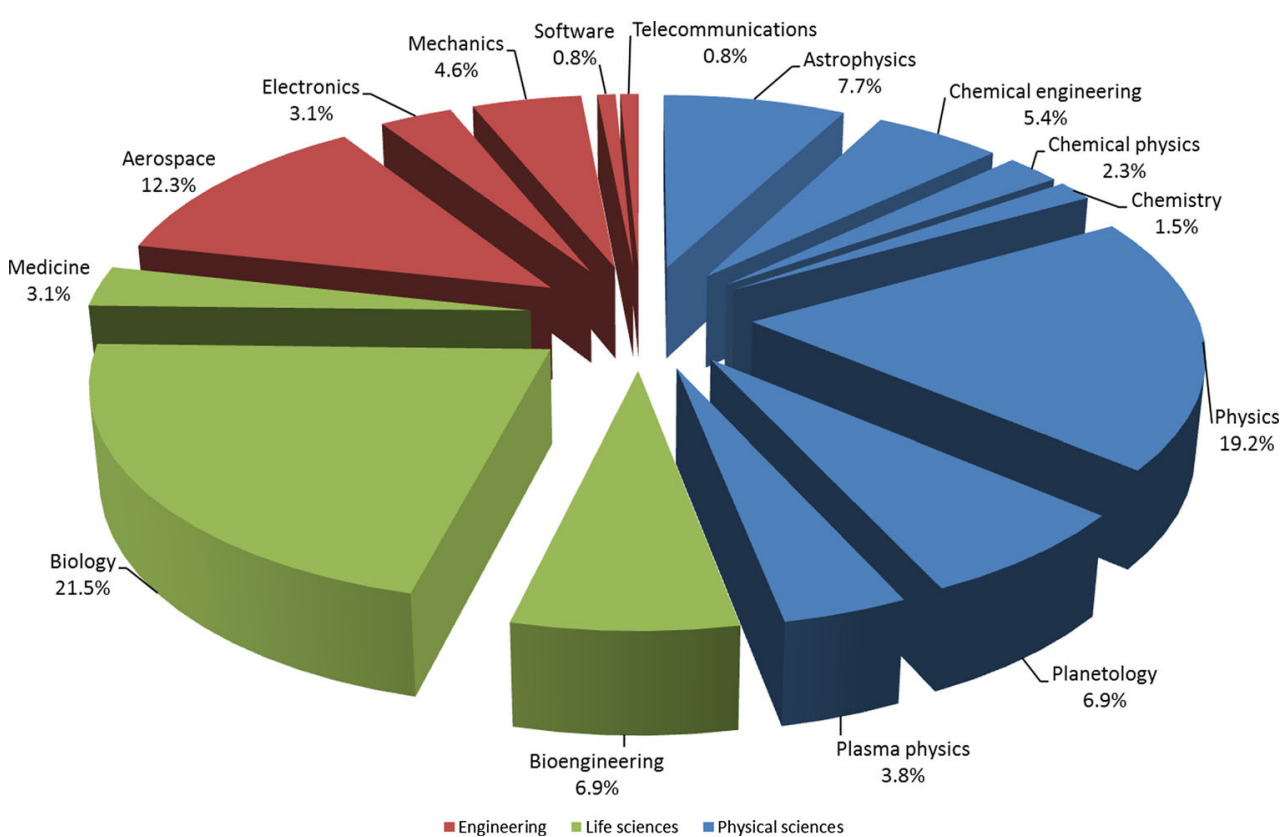


Fig. 4 Distribution of experiment fields

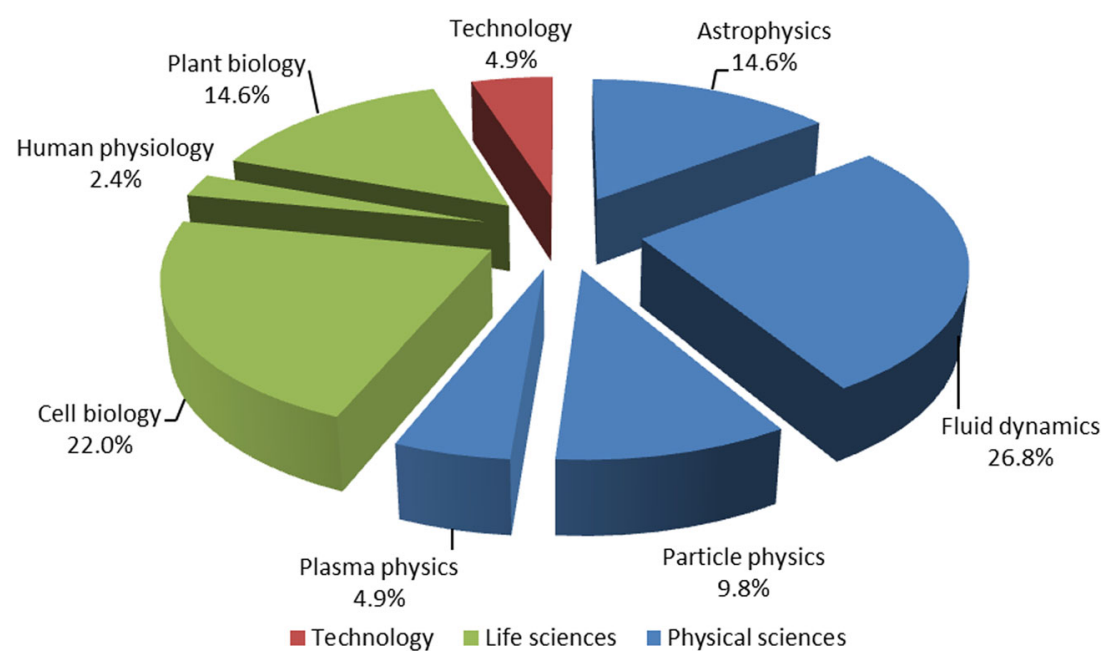

postures and movements in microgravity. To obtain the naturally adopted posture of the human body, nine test subjects had to follow one of two predefined commands, "stretch" or "crouch", while free floating in the microgravity phase. After performing the task, the test subject had to relax and avoid any intentional movement of the body (Fig. 6). This phase was intended to show how the body slowly resumes its neutral position. To reduce the environmental distractions, the test subjects were provided with blindfolds and headphones. Each test subject underwent the same experiment underwater, allowing for comparable data sets in both environments. A wide variety of neutral postures could be observed between different subjects and between successive sessions of single subjects. Most of the subjects were able to fulfill the commands and defined exercises, but did

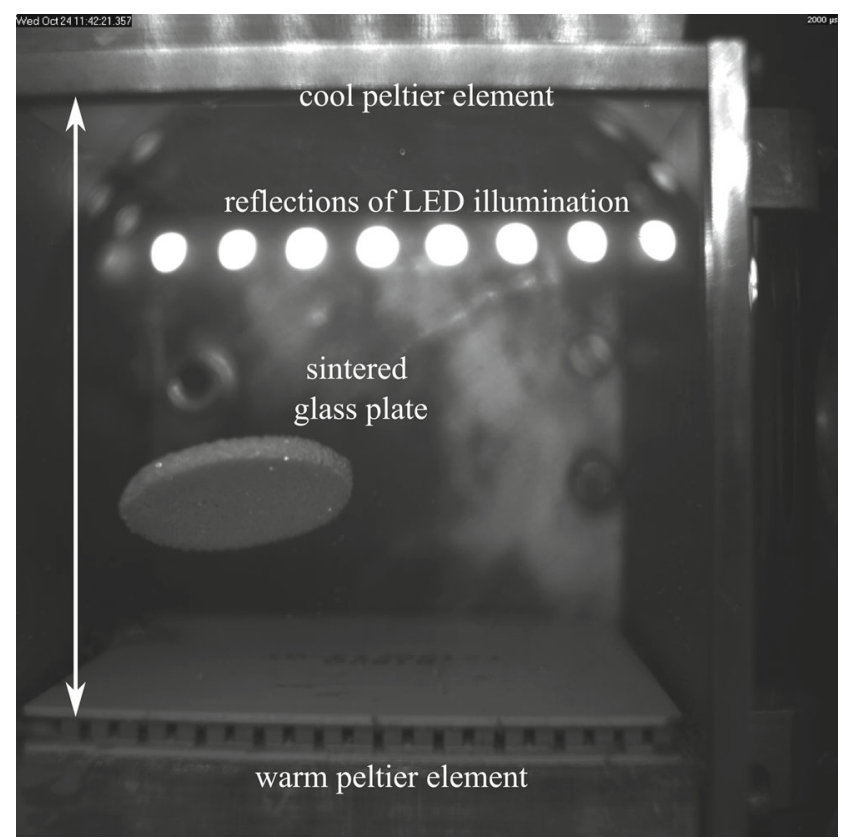

Fig. 5 Levitated sintered glass plate (Picture: Dustbrothers team) 
Fig. 6 Stable posture of test subject in microgravity with grid (Picture: Hydronauts2fly team)

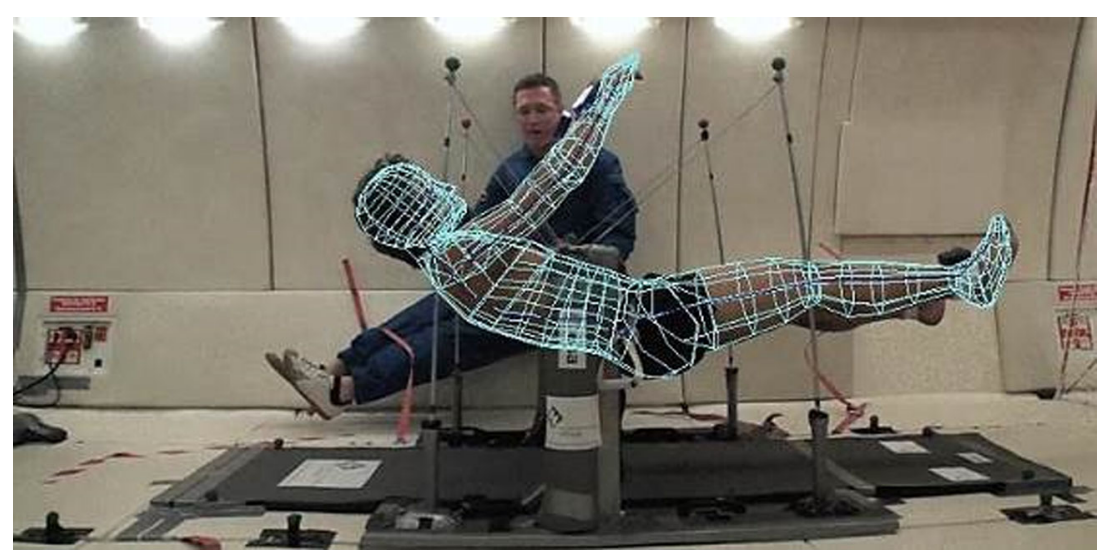

not resume a fully extended body position at the end of a cycle. This phenomenon is believed to be caused by the human body's own inertia. Test subjects adapted to similar postures in both test environments, even though the available experiment time on parabolic flights was much shorter than underwater. It was concluded that the drag influence of water on the human body's inertia was much weaker than expected. It is primarily the individual muscular system which is driving the body's adaptation to their neutral body posture. However, the buoyancy of air-filled lungs needs to be taken into account when performing underwater tests. Knowledge of the human posture is useful for the design of future spacecraft interiors and space suits.

\section{“Drop Your Thesis!” Programme}

The "Drop Your Thesis!" programme offers university students, from Bachelor to $\mathrm{PhD}$, the opportunity to launch their experiment five times in the Bremen Drop Tower. This facility provides around $4.7 \mathrm{~s}$ of microgravity in drop mode and $9.3 \mathrm{~s}$ of microgravity in catapult mode, with a residual gravity on the order of $10^{-6} \mathrm{~g}$ (von Kampen et al. 2006). Between the launches, the students can easily adjust or improve their experiment set-up.

\section{Technology: New Docking System for Spacecrafts}

The FELDs team, from the University of Padova ${ }^{9}$ in Italy, performed an experiment to test a new docking system that uses a tether to connect two spacecraft under microgravity conditions. The docking was performed by ejecting a spherical probe towards its receiver, which attracted the probe with a strong electromagnet. The receiver was reeled in by retracting the tether (Fig. 7).The aim of this experiment was to overcome drawbacks of traditional docking systems by relaxing the accurate orbital control requirements of the

\footnotetext{
${ }^{9}$ www.unipd.it
}

docking manoeuvre by allowing final trajectory adjustments to be performed passively by an induced magnetic field.

This experiment was the first of its kind that tested and validated this concept in microgravity conditions. The obtained data allowed the team to create a theoretical model of the impact forces and the response of the system and a mathematical description of the motion of the tethered system (Petrillo et al. 2015). The results of this experiment served both as a proof of concept for the FELDs technology and as a study of the tethered probe dynamics. The
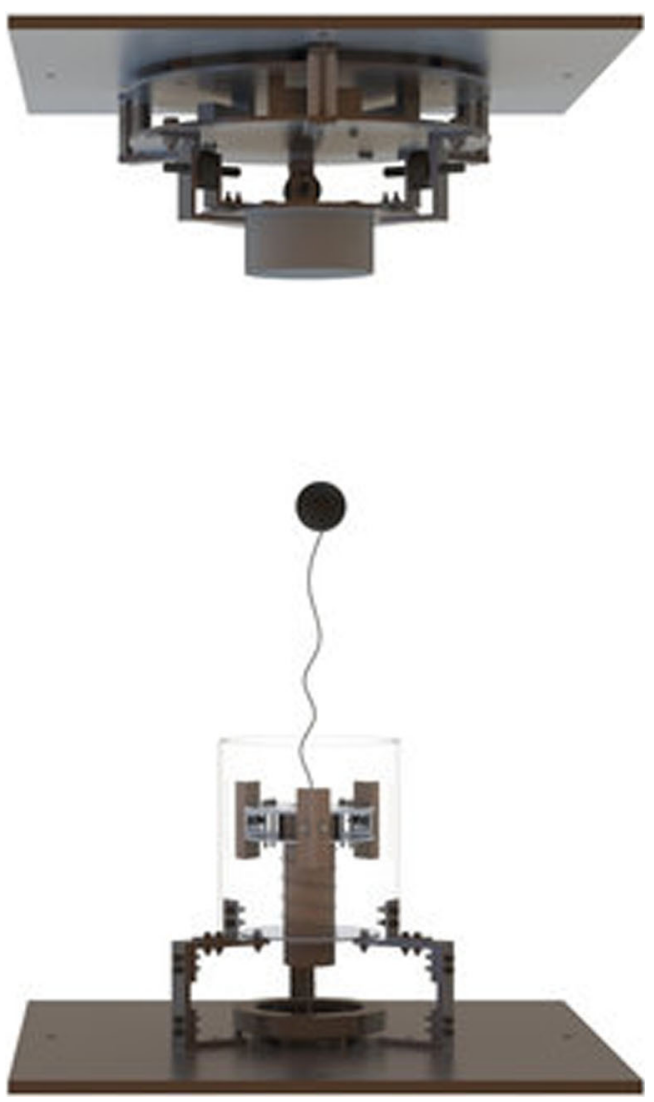

Fig. 7 Docking system using a tether tested in microgravity (Picture: FELDs team) 


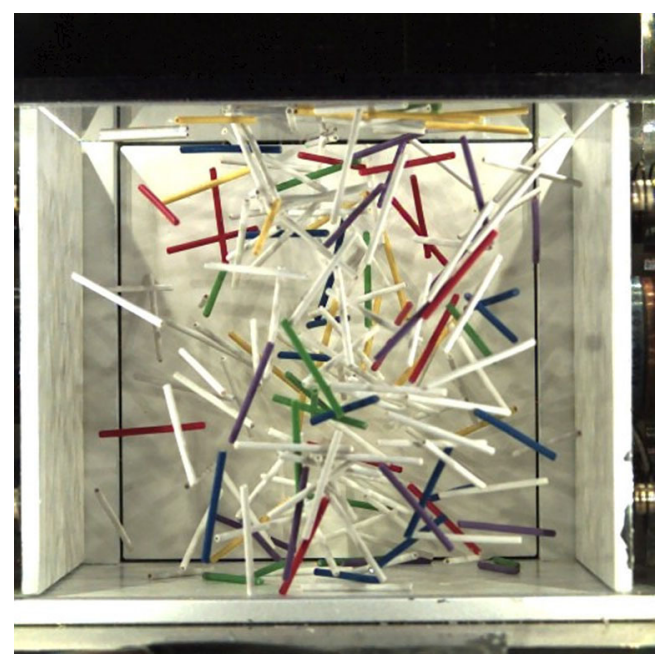

Fig. 8 Granular material in microgravity inside a container with three independently controllable vibrating walls (Picture: GAGaDropT team)

usefulness and limits of the capture effect given by the electromagnet on the target system were characterized from the data of the successful attempts and compared with the theoretical model. The release system proved to be critical; a better solution, with negligible friction, will need to be implemented in future tethered docking solutions. The data obtained from this experiment is a first step towards full, space-viable, implementation of a flexible docking system.

\section{Particle Physics: Aggregation Phenomena, Granular} Systems

The GAGaDropT team, from the Otto-von-Guericke University Magdeburg ${ }^{10}$ in Germany, performed an experiment to study the behaviour of granular gases in microgravity conditions. The experiment was a follow-up of the sounding rocket experiment in the GAGa REXUS campaign (Harth et al. 2013). The experiment set-up consisted of a container, with three independently controllable vibrating walls, containing rod shaped granular material (Fig. 8). Several content materials were tested, while position, orientation, and velocity data were obtained in three dimensions. The students developed an excitation protocol to obtain a homogeneous distribution that depended on the initial excitation and the positioning of the granular material. The outcomes from this experiment are now being used in further follow-up research, the results of which will improve the understanding of the fundamental principles of granular dynamics.

\footnotetext{
${ }^{10}$ www.ovgu.de
}

\section{“Spin Your Thesis!" Programme}

The "Spin Your Thesis!" programme offers university students, from Bachelor to $\mathrm{PhD}$, to perform experiments at the ESTEC Large Diameter Centrifuge (LDC). This facility can achieve centrifugal accelerations up to 20 times Earth's gravity $(20 \mathrm{~g})$ and can hold up to six gondolas on the 8 meter arms. Students can use the centrifuge for 2.5 days with the flexibility to set the desired acceleration levels while also allowing for easy adjustment of their experiment set-up and procedure at any time during the course of the experiment execution.

\section{Cell Biology: Nanotechnology Systems as Regenerative Medicine}

The Osteo team, from the Istituto Italiano di Tecnologia ${ }^{11}$ in Italy, studied nanotechnology systems for application in the field of regenerative medicine. Mesenchymal stem cells are important in the process of tissue repair and regeneration in organisms. Biological organisms are exposed to different factors that influence their proliferation and differentiation. Nanomaterials can be used to influence these key factors to manipulate and control the cell's function in tissue regeneration (Ferreira et al. 2015). In this experiment, the effects of hypergravity (as mechanical stimulus) and Barium Titanate Nano Particles (BTNPs) on the osteogenesis of rat mesenchymal stem cells were tested. The team investigated whether the nanomaterial would be better internalized into the cellular system under hypergravity conditions.

Samples underwent three hours of treatment at $20 \mathrm{~g}$ in the LDC, in the presence or absence of barium titanate nanoparticles. Afterwards, they were analysed and compared with control samples at $1 \mathrm{~g}$ (Fig. 9). Cells that underwent hypergravity showed morphology changes and an increment of cell defence mechanisms. Moreover, the cells incorporated the nanoparticles at a higher extent during hypergravity conditions, and therefore showed increased promotion of osteogenesis (Rocca et al. 2015). These promising results could be very useful in the design of new approaches for bone regeneration and in drug delivery systems of in vitro cellular constructs.

\section{Fluid Dynamics: Bubble Dynamics}

The Bubble Movers team, from the Universitat Politecnica de Catalunya ${ }^{12}$ in Spain, studied the dynamics of bubbles rising in a tank of liquid under acoustic waves in hypergravity conditions. The experiment set-up consisted of a test cell filled with distilled water, a bubble injection system, two

\footnotetext{
${ }^{11}$ www.iit.it

${ }^{12} \mathrm{http}: / /$ www.upc.edu
} 
Fig. 9 Hypergravity effect on mesenchymal stem cells with and without Barium Titanate Nano Particles (BTNPs) (Picture: Osteo team)

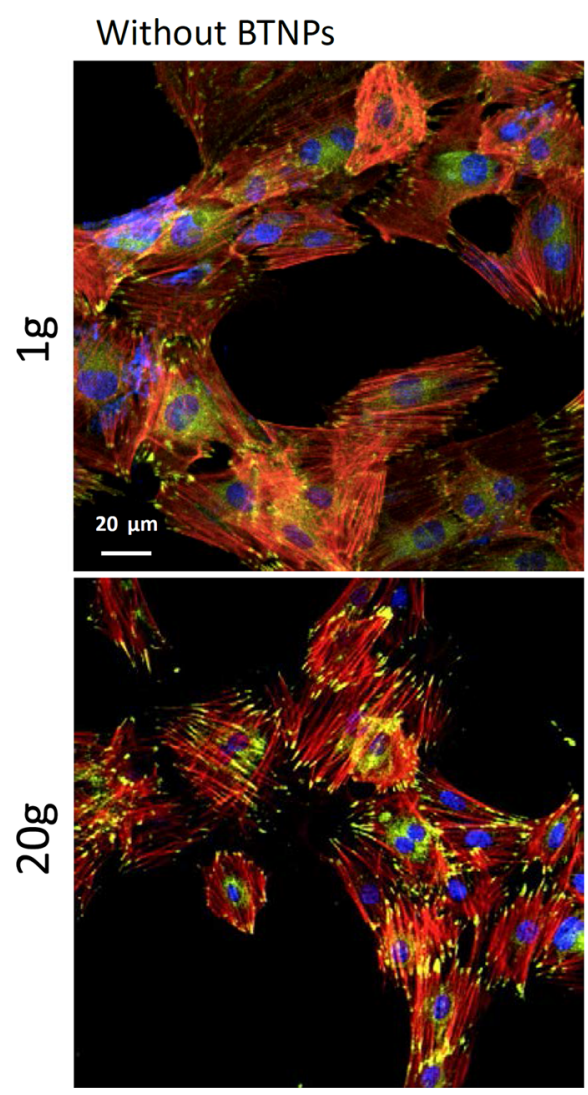

With BTNPS

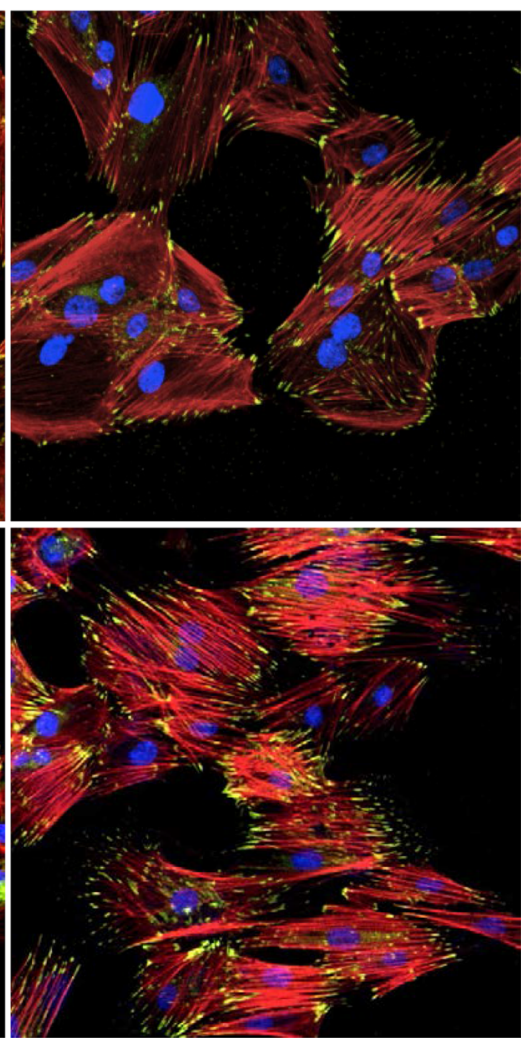

piezoceramic transducers to generate the acoustic waves, and a data acquisition system to make high-speed videos of rising bubbles (Fig. 10).

Throughout the data collection, the students observed unique features of the bubbles. With a fixed nozzle diameter, the bubble size was determined by the balance between gravitational and surface tension forces. In hypergravity, the periodic rising trajectories of the bubbles had larger frequencies and smaller amplitudes. A vorticity was produced at the bubble surface that grew with increasing gravity and generated a wake instability that destabilized the flow (Suñol and González-Cinca 2015). Moreover, the large injection flow rates generated bubble trains. Due to the wake of the leading bubbles, trailing bubbles presented larger terminal velocities, compared to isolated bubbles. The acoustic waves caused different effects on the bubbles, including lower detachment diameters as the acoustic pressure increased, bubble levitation, and interaction between bubbles or cavitation.

\section{Impact on Students' Studies and Career Paths}

Participation in these three ESA Education programmes enables university students to work, often for the first time, in a team, on a hands-on project, providing them with the opportunity to gain important experience and develop skills in preparation for their future career. ESA's Education

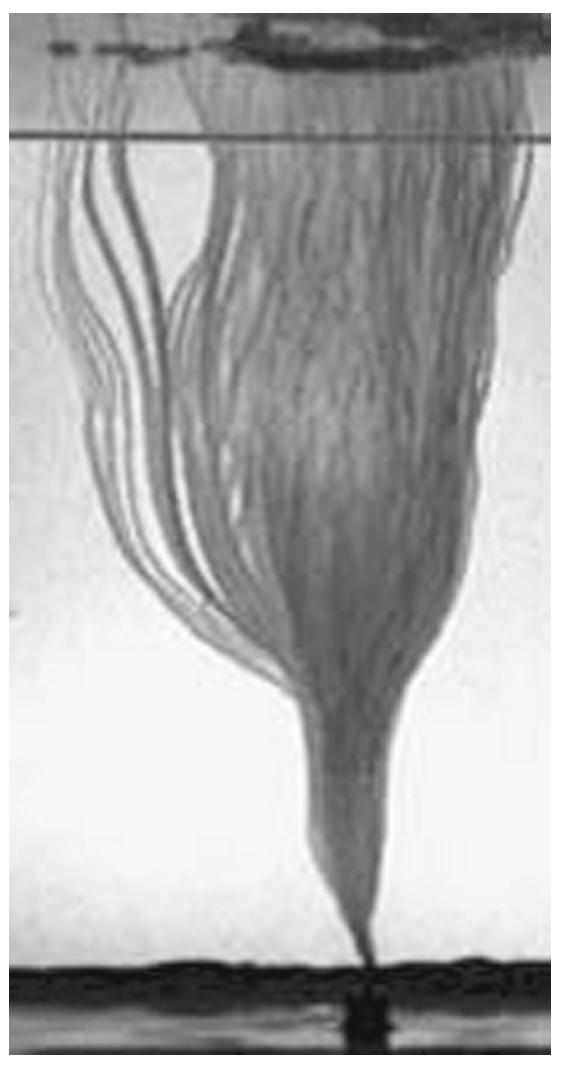

Fig. 10 Image obtained overlapping the frames of each video of rising bubbles (Picture: Bubble Movers team) 


\begin{tabular}{|c|c|c|c|c|c|c|}
\hline & \\
\hline & High & $\begin{array}{c}\text { Medium } \\
\text { High }\end{array}$ & Medium & $\begin{array}{c}\text { Medium } \\
\text { Low }\end{array}$ & Low & N/A \\
\hline Practical application of knowledge (building/ testing) & $62.3 \%$ & $27.9 \%$ & $4.9 \%$ & $1.6 \%$ & $1.6 \%$ & $1.6 \%$ \\
\hline Access to professional facilities/ testing environment & $55.7 \%$ & $29.5 \%$ & $8.2 \%$ & $1.6 \%$ & $3.3 \%$ & $1.6 \%$ \\
\hline Practical application of knowledge (design) & $51.6 \%$ & $38.7 \%$ & $1.6 \%$ & $3.2 \%$ & $1.6 \%$ & $3.2 \%$ \\
\hline Self learning & $48.4 \%$ & $30.7 \%$ & $19.3 \%$ & $0.0 \%$ & $1.6 \%$ & $0.0 \%$ \\
\hline Contact with experts & $47.5 \%$ & $32.8 \%$ & $14.8 \%$ & $3.3 \%$ & $1.6 \%$ & $0.0 \%$ \\
\hline Writing reports & $35.5 \%$ & $24.2 \%$ & $27.4 \%$ & $6.5 \%$ & $1.6 \%$ & $4.8 \%$ \\
\hline Written information (user manuals, templates, guidelines) & $27.4 \%$ & $35.5 \%$ & $22.6 \%$ & $8.1 \%$ & $1.6 \%$ & $4.8 \%$ \\
\hline Contact with students from other universities & $12.9 \%$ & $21.0 \%$ & $30.7 \%$ & $11.3 \%$ & $16.1 \%$ & $8.1 \%$ \\
\hline
\end{tabular}

Fig. 11 Usefulness of the learning methods as experienced by the respondents. Percentages represent how many students voted for each option

Office conducted a survey from June to December 2014 using an Internet-based tool (SurveyMonkey ${ }^{13}$ ) to evaluate the benefits of these hands-on programmes in the view of the students who participated in the past. The following factors were considered: the outcome of the student project, how the student's participation increased their skills and knowledge, whether the participation in these programmes created career opportunities, and whether it impacted their professional life. The survey was conducted confidentially with the possibility to submit voluntary answers to all questions. 84 students from the 31 different teams that participated between 2009 and 2013 were contacted to fill in the questionnaire. 63 students completed the survey and replies were gathered from students from all participating teams.

Approximately $55 \%$ of the respondents were still studying, around $12 \%$ at Bachelor, $5 \%$ at Master and $38 \%$ at $\mathrm{PhD}$ level while $45 \%$ were working. Seventy-four percent of the respondents stated that their participation increased their interest in choosing a space-related career. Interestingly, all of the working respondents are currently employed in a STEM field and $39 \%$ have a space-related job. Sixtyseven percent of the respondents found their participation relevant to their career, $31 \%$ of the respondents said that the programme was a springboard for their career, and $23 \%$ said it will be at some point. In order to quantitatively evaluate the outcome of the various student projects, and to assess the quality of their obtained data, the number of these projects published in renowned scientific or technical journals and presented at conferences has been estimated. In December 2014, $87 \%$ of the teams confirmed that they presented or published their experiment results. $74 \%$ of the teams presented their results at a conference, 9 presentations in life sciences and 27 in physical sciences have been listed. Forty-five percent of the teams published a paper in a renowned journal, 8 papers in life sciences and 13 in physical sciences have been identified. Additionally, several Bachelor, Master and $\mathrm{PhD}$ theses include results obtained through these student projects.

\footnotetext{
${ }^{13}$ www.surveymonkey.net
}

A few of the most notable conferences listed among the responses included: the ELGRA Biennial Symposium ${ }^{14}$, the COSPAR Scientific Assembly ${ }^{15}$, and the International Astronautical Conference ${ }^{16}$.

Results of the student projects were published in many different journals, including Microgravity Science and Technology, Granular Matter, Journal of Physics: Conference Series, Acta Astronautica, and Astronomy and Astrophysics. Some of these publications included the results of multiple projects. Additional papers were submitted in 2015 and are currently under review.

In addition to increasing visibility in the academic realm through presentations and publications, students also promoted their project and the ESA Education programmes through public outreach; some teams, for example, were featured in local newspapers, and were interviewed on radio and television, others were recognized by international awards.

Most of the learning methods offered in the frame of the three hands-on programmes were found highly useful by respondents (Fig. 11). In particular, the practical application of knowledge for designing, building, and testing their experiments. Respondents also appreciated the unique access to professional facilities and the contact with experts provided through the programmes. Moreover, the ESA provided resources on learning how to write a report and how to use written information, such as user manuals, templates, and guidelines, were also found useful. Finally, the opportunity for students to make contact with students from other European universities was marked favourably by a majority of respondents.

\section{Conclusions}

Since 2009, ESA's Education Office has organized 16 gravity-related student experiment campaigns in the frame of the "Fly Your Thesis!", "Drop Your Thesis!" and "Spin

\footnotetext{
${ }^{14}$ http://www.elgra.org/

${ }^{15}$ https://www.cospar-assembly.org/

${ }^{16}$ http://www.iafastro.org/events/iac/
} 
Your Thesis!" programmes. These campaigns involved 130 university students from 42 teams coming from 39 different universities across Europe and Canada. These programmes increased the interest of many participants in space-related careers by allowing them to work in a team, on a spacerelated project and gain practical experience. Quite importantly, the benefits deriving from the participation in these programmes extend far beyond the primary educational objectives, allowing students to develop new skills and to learn first-hand expertise from the mentors and the specialists supporting their project. Recently, the ESA Education Office created the ESA Academy, which includes in addition to the hands-on activities a training activity to facilitate the transfer of ESA's knowledge and expertise to university students. Through the ESA Academy, the ESA Education Office will continue to provide growth and learning opportunities for university students and facilitate their transition in becoming young professionals in the STEM fields.

Acknowledgments The success of these three hands-on programmes is based on an excellent collaboration between colleagues from ESA and from all organizations which are participating in these programmes, on the high motivation of the participating students, and on the support that universities offer to their student teams to participate in the programmes of the ESA Education Office.

Students from the following teams have contributed to this manuscript and the authors would like to thank them for their inputs: Dustbrothers team from the University of Duisburg-Essen (Germany), Hydronauts2fly team from the Technical University of Munich (Germany), FELDs team from the University of Padova (Italy), GAGaDropT team from the Otto-von-Guericke University Magdeburg (Germany), Osteo team from the Istituto Italiano di Tecnologia (Italy), Bubble Movers team from the Universitat Politecnica de Catalunya (Spain). The authors also wish to thank all colleagues from Novespace, ZARM FAB, ELGRA and from ESA as well as all the students and the personnel from their universities in Europe and Canada that helped them to develop their experiments and perform their investigations.

Open Access This article is distributed under the terms of the Creative Commons Attribution 4.0 International License (http:// creativecommons.org/licenses/by/4.0/), which permits unrestricted use, distribution, and reproduction in any medium, provided you give appropriate credit to the original author(s) and the source, provide a link to the Creative Commons license, and indicate if changes were made.

\section{References}

Bruzzi, D., Tortora, P., Giulietti, F., Galeone, P., De Luca, A.: The ESEO Development: Merging Technical with Educational Challenges. The 4S Symposium, Majorca, Spain (2014)
Callens, N., Dannenberg, K., Fittock, M., Inga, M., Kinnaird, A., Persson, O., Roth, M., Schmidt, A., Siegl, M.: REXUS/BEXUS Rocket and Balloon Experiments for University Students. 21th ESA Symposium on European Rocket and Balloon Programmes and Related Research, Thun Switzerland (2013)

Callens, N., Galeone, P.: Getting 'Space' experience on Earth - education activities for university students. ESA Bull. 157 (2014)

Callens, N., Ventura-Traveset, J., de Lophem, J., Lopez, T.-L., de Echazarreta, C., Pletser, V., van Loon, J.: Fly Your Thesis! - an astronaut experience - ESA Parabolic Flights opportunities for university students in collaboration with ELGRA. ELGRA Biennial Symposium, Bonn, Germany. ELGRA News 26, 81 (2009)

Emma, F., Ventura-Traveset, J., Walker, R., Page, H., Lopez de Echazarreta, C., de Lophem, T.-L., Cross, M., Berglund, P., Nikolaidis, P.: ESA Hands-on Project Education Strategy. 59Th International Astronautical Federation Congress IAC-08-E1.1.2, Glasgow, Scotland (2008)

Ferreira, T.H., Rocca, A., Marino, A., Mattoli, V., de Sousa, M.B., Ciofani, G.: Evaluation of the effects of boron nitride nanotubes functionalized with gum arabic on the differentiation of rat mesenchymal stem cells. Royal Society of Chemistry 5, 45431 (2015)

Galeone, P., Vanreusel, J., Gulino, M., Sagath, D., Gupta, V.: The Fly Your Satellite! Programme of the ESA Education Office. The 4S Symposium, Majorca, Spain (2014)

Harth, K., Kornek, U., Trittel, T., Strachauer, U., Home, S., Will, K., Stannarius, R.: Granular gases of rod-shaped grains in microgravity. Phys. Rev. Lett. 110, 144102 (2013)

Küpper, M., Dürmann, C., de Beule, C., Wurm, G.: Propulsion of porous plates in thin atmospheres by temperature fields - experiments on parabolic flights (2013)

Petrillo, D., Buonomo, M., Cavinato, A., Chiariotti, F., Gaino, M., Branz, F., Mantellato, R., Olivieri, L., Sansone, F., Francesconi, A., Borst, G.: Flexible Electromagnetic Leash Docking System (FELDs) Experiment from Design to Microgravity Testing. In: 66Th International Astronautical Congress, IAC-15, E2, 3YPVF.4, 11, x28726, Jerusalem, Israel (2015)

Pletser, V., Harrod, J.: The science of gravity; a new era of ESA experiments on parabolic flights. ESA Bull. 160, 22-33 (2014)

Pletser, V., Rouguette, S., Friedrich, U., Clervey, J., Gharib, T., Gai, F., Mora, C.: European parabolic flight campaigns with Airbus A300 ZERO-g: looking back at the A300 and looking forward to the A310. Adv. Space Res., 1003-1013 (2015)

Rocca, A., Marino, A., Rocca, V., Moscato, S., De Vito, G., Piazza, V., Mazzolai, V., Mattoli, V., Ngo-Anhg, T.J., Ciofani, G.: Barium titanate nanoparticles and hypergravity stimulation improve differentiation of msenchymal stem cells into osteoblasts. Int. J. Nanomedicine 10(8), 433-445 (2015)

Suñol, F., González-Cinca, R.: Effects of gravity level on bubble formation and rise in low-viscosity liquids. Phys. Rev. E91, 053009 (2015)

van Loon, J., Krause, J., Cunha, H., Goncalves, J., Almeida, H., Schiller, P.: The Large Diameter Centrifuge, LDC, for life and physical sciences and technology. In: Proceedings of the 'Life in Space for Life on Earth Symposium', Angers, France ESA SP-663 (2008)

von Kampen, P., Kaczmarczik, U., Rath, H.J.: The new Drop Tower catapult system. Acta Astronaut., 59 (2006) 